\title{
Propágulos de Rhizophora mangle (Rhizophoraceae) barrenados por Coccotrypes rhizophorae (Coleoptera: Curculionidae) en el manglar de Tumilco, Veracruz, México
}

\author{
Alejandro Arturo Martínez-Zacarías, Ivette Alicia Chamorro-Florescano*, \\ Juan Manuel Pech-Canché, José Luis Alanís-Méndez \& Agustín de Jesús Basáñez-Muñoz \\ Facultad de Ciencias Biológicas y Agropecuarias, Universidad Veracruzana, Campus Tuxpan. Carretera Tuxpan a \\ Tampico km.7.5, C.P. 92850 Tuxpan Veracruz, México; azacariasm@hotmail.com, ivette.chamorro@gmail.com, \\ jmpech@gmail.com, lalanis@uv.mx, abasanez@uv.mx \\ * Correspondencia
}

Recibido 04-X-2016. Corregido 04-V-2017. Aceptado 02-VI-2017.

\begin{abstract}
Propagules of Rhizophora mangle (Rhizophoraceae) bored by Coccotrypes rhizophorae (Coleoptera: Curculionidae) in the Tumilco mangrove, Veracruz, México. Mangrove species propagules represent a particular survival and dispersal strategy. Unfortunately, some reports have stated that Rhizophora mangle propagules are frequently attacked by Coleoptera and Lepidoptera before and after their dispersion period. The beetle species Coccotrypes rhizophorae is an obligate parasite that can cause mortality in propagules and seedlings of $R$. mangle, and may modify the structure of its populations. Because of the importance on this mangrove species, survival and increasing human impacts in the area, this study evaluated the presence of propagules with C. rhizophorae boring evidence in the Tumilco mangrove, in Veracruz State, Mexico. For this, three sampling areas were established: 1) mangrove core of $R$. mangle, 2) most impacted edge of an unpaved road with $R$. mangle and A. germinans, and 3) edge with Typha latifolia and $R$. mangle; in each area, three $25 \times 25$ $\mathrm{m}$ random plots were analyzed. The number of bored propagules per site was recorded every 15 days over a sampling period of one year $(n=567)$ from January to December 2012. We also observed the damaged part of the propagule (basal, central, apical) and the number of live and dead bored propagules per season, and compared results for different seasons in the area: rainy, cold northerly fronts or "norte" and dry. Insects were identified by an expert. Our results showed that a higher number of bored propagules was observed during the dry season, mainly in zones 2 and 3. Survival of bored propagules was higher when the perforation occurred in the basal part of the plant, and during the dry and norte seasons. Study zone 2 in particular presented a higher number of live propagules when the damage occurred in the basal part of the propagule. In all three zones, mortality was higher when the damage was apical. Zones 1 and 3 presented higher propagule survival during the dry season, while survival in zone 2 was higher during the rainy and norte seasons. The number of propagules decreased with rising flooding levels. The results showed that $C$. rhizophorae has a wide distribution in the Tumilco mangrove, but the mortality of $R$. mangle propagules is influenced by the part of the propagule that suffers damage, as well as by the season of the year and the characteristics of the area in which the infestation occurs. Futher studies are required to evaluate the impact of propagule mortality on the population structure of $R$. mangle. Rev. Biol. Trop. 65 (3): 1120-1128. Epub 2017 September 01.
\end{abstract}

Key words: survival, beetle, predation, red mangrove, mortality.

La depredación de propágulos y plántulas en los bosques de manglar por artrópodos, como lepidópteros y coleópteros, puede reducir el número y la calidad de las plántulas durante la fase de establecimiento (Onuf, Teal, \& Valiela, 1977; Feller \& Mathis, 1997; Cannicci et al., 2008). Los insectos de la familia Curculionidae son comúnmente asociados como parte de las amenazas que sufren las plantaciones forestales. Se caracterizan por ser especies pequeñas ( $5 \mathrm{~mm}$ máximo) que barrenan ramas, troncos, raíces y plántulas de una gran variedad 
de árboles, construyen galerías dentro del hospedero, las cuales pueden ser de diferentes dimensiones, e incluso mantener una variada distribución, destruyendo tejido que puede ser primordial para el desarrollo, establecimiento y supervivencia de las plantas (Wood, Stevens, \& Lezama, 1991; Sousa, Kennedy, \& Mitchell, 2003a; Sousa, Quek, \& Mithell, 2003b; Devlin, 2004).

Coccotrypes rhizophorae Hopkins es un curculiónido identificado como un parásito obligado que puede dañar las raíces adventicias y causar la muerte de propágulos y plántulas de Rhizophora mangle L.; este insecto depende de su hospedero para vivir los diferentes estados de su vida, únicamente las hembras son las que migran para ocupar otros hospederos, una relación planta-animal que puede influir en la resiliencia de los bosques de mangle (Woodruff, 1970; Devlin, 2004; Menéndez, Guzmán, \& Núñez, 2006; Cannicci et al., 2008). C. rhizophorae se ha registrado afectando manglares de diferentes países, como Estados Unidos, Cuba, Belice, Panamá, Costa Rica, Perú, México, Malasia, Java, Sumatra, India, Galápagos, Samoa y Singapur. En algunos casos, se menciona que puede existir un equilibrio entre las poblaciones de insectos y la conservación del manglar, con una tendencia por parte de los insectos a aumentar su abundancia, cuando se produce un estado de estrés en las plantas originado por una red de factores ambientales (Rabinowitz, 1977; Maiti \& Saha, 1986; Murphy, 1990; Feller \& Mathis, 1997; Bright \& Peck, 1998; Castillo, 2001; Sousa et al., 2003b; Vázquez-Moreno, RodríguezPérez, \& Zorrilla, 2003; Menéndez et al., 2006; Devlin, 2004).

Factores ambientales tales como salinidad, nivel de inundación, humedad e iluminación potencialmente pueden influir en el estrés y la infestación de propágulos y plántulas en los manglares (Onuf et al., 1977; Sousa et al., 2003b; Devlin, 2004; Krauss et al., 2008). Por ejemplo, muchas especies de escolítidos son atraídos por la luz, por lo que es más frecuente su presencia en áreas alteradas o zonas donde se generan claros por caída de árboles (Sánchez-Martínez \& Wagner, 2002); sin embargo, algunos estudios han encontrado que las zonas cerradas dentro del manglar, presentan un mayor número de propágulos depredados que las zonas abiertas (Sousa et al., 2003b). La afectación del escarabajo herbívoro $C$. rhizophorae sobre $R$. mangle es importante antes y después de la dispersión de los propágulos (más frecuentemente en los propágulos abortados); sin embargo, la relación tamaño y el daño generado por los insectos sobre el propágulo ha sido poco estudiada (Sousa et al., 2003a; Souza \& Sampaio, 2011).

Este trabajo tuvo como principal objetivo conocer la frecuencia de propágulos de $R$. mangle barrenados por $C$. rhizophorae a lo largo de un año, así como evaluar la supervivencia de los propágulos barrenados en el sitio Ramsar, Manglares y Humedales de Tuxpan, ubicado en Tumilco, Veracruz, México.

\section{MATERIALES Y MÉTODOS}

Los Manglares y Humedales de Tuxpan (Sitio Ramsar 1602) se encuentran divididos por el río Tuxpan, al norte se ubican los manglares de la Laguna de Tampamachoco, y al sur los manglares y humedales asociados a los esteros de Tumilco y Jácome en el estado de Veracruz, México. Este manglar, con una extensión de 6870 hectáreas, cuenta con cuatro de las seis especies de mangle en México (Rodríguez-Zúñiga et al., 2013). En los alrededores de los manglares y humedales de Tuxpan, el cambio de uso del suelo hacia actividades agropecuarias, urbanas e industriales, se ha incrementado notablemente en los últimos años (Basáñez-Muñoz, 2005).

Para el estudio de campo se establecieron tres zonas de muestreo en el manglar de Tumilco, a) zona 1, en la zona núcleo del manglar, que mantiene un dosel más cerrado predominado por $R$. mangle $\left(20^{\circ} 55^{\prime} 35.1\right.$ ' N \& 097²0'58.5" $\mathrm{W})$; b) zona 2, en el borde del manglar, al lado de la carretera de terracería de la comunidad de Tumilco, que corresponde al lugar más alterado, con presencia de $R$. mangle y $A$. germinan (2055’33.8“ N \& 097²0’09.6“ W); y c) zona 
3 , borde del manglar al lado de una zona herbácea de Typha latifolia con predominio de $R$. mangle (2054 46.5“ $\left.\mathrm{N} \& 097^{\circ} 19^{\prime} 17.2^{\prime \prime} \mathrm{W}\right)$. En las tres zonas, se establecieron tres cuadrantes de manera aleatoria de $25 \mathrm{~m}$ por $25 \mathrm{~m}$.

En cada cuadrante, se registraron los propágulos de $R$. mangle barrenados por $C$. rhizophorae dos veces por mes durante un año, de enero a diciembre de 2012, los escolítidos fueron identificados por el Dr. Thomas H. Atkinson. En agosto no hubo registros debido a una inundación provocada por la tormenta Ernesto que azotó la zona ese año. Se catalogaron tres temporadas reconocidas para la región: lluvias de julio a octubre, nortes de noviembre a febrero y secas de marzo a junio (Toledo-Ocampo, 2005). Cada propágulo barrenado encontrado dentro de los cuadrantes, fue marcado con cinta flaguin para individualizarlo, de tal forma que se identificó cada nuevo propágulo a lo largo del año. En los propágulos barrenados se llevó a cabo un registro con los siguientes parámetros: 1) ubicación del daño (basal, medio o apical), se refiere al lugar en que se encontró la perforación, identificada por la presencia de "aserrín" y el hueco de entrada de los escolítidos; 2) también se determinó el tamaño del propágulo (largo total en $\mathrm{cm}$ ), y 3 ) la condición del propágulo (vivo o muerto).

De la misma forma, se obtuvieron registros mensuales de parámetros fisicoquímicos (temperatura, salinidad, $\mathrm{pH}$, precipitación $\mathrm{y}$ humedad) que fueron proporcionados por la Comisión Nacional del Agua (CONAGUA) para la región de Tuxpan, Veracruz. El nivel de inundación superficial se midió por cuadrante, cada quince días con una regla de plástico graduada en milímetros (Rico-Gray \& Palacios-Ríos, 1996).

Para el análisis de los datos, se realizó un modelo lineal generalizado (GLM) con distribución Poisson con liga identity para evaluar si el número de propágulos barrenados por $C$. rhizophorae depende de la temporada (lluvias, nortes y secas) y de las zonas muestreadas (1,2 y 3), en el manglar de Tumilco.

Un GLM con distribución binomial con liga logit se usó para determinar si la supervivencia de los propágulos barrenados depende de la zona (1, 2 y 3), posición del daño (basal, medio o apical) y temporada (lluvias, nortes y secas), así como la interacción de estas variables. Además, se incluyó el largo total de cada propágulo como una covariable en el modelo. La supervivencia se calculó con la variable respuesta obtenida con la función cbind, mediante un doble vector que contiene el número de propágulos vivos y muertos (Crawley, 2013). Los resultados se presentan como promedio \pm error estándar.

En ambos GLM, el modelo mínimo adecuado se seleccionó usando el método stepwise backward y comparando los valores de Akaike Information Criteria (AIC); así el modelo con el más bajo AIC se consideró el más adecuado (Crawley, 2013).

Para los datos de parámetros fisicoquímicos, se utilizó una regresión para evaluar cada variable independiente con la variable dependiente, número de propágulos. Todos los análisis fueron conducidos en el paquete estadístico R 2.1.7 (R Development Core Team, 2014).

\section{RESULTADOS}

El análisis reflejó que la temporada tuvo un efecto significativo sobre el número de propágulos barrenados por $C$. rhizophorae $\left(\chi^{2}\right.$ $=4.02, \mathrm{p}=0.019)$. La temporada de secas tuvo mayor promedio de propágulos barrenados $(10 \pm 1.98)$, descendiendo en la temporada de nortes $(22.67 \pm 4.58)$ y lluvias $(30.33 \pm 9.05)$. La interacción entre zona muestreada y temporada también influyó de manera significativa en el número de propágulos registrados $\left(\chi^{2}=\right.$ $17.86, \mathrm{p}=0.001)$; la zona 1 que correspondió al núcleo del manglar, mantuvo un número de propágulos barrenados similar en las tres temporadas, pero bajo en frecuencia, mientras que la zona 2 (al lado de la carretera) y 3 (al lado del Typha) aumentaron gradualmente el número de propágulos barrenados en la temporada de nortes y secas (Fig. 1).

Al evaluar la supervivencia de los propágulos, el modelo mostró que los predictores que influyeron de manera significativa fueron 


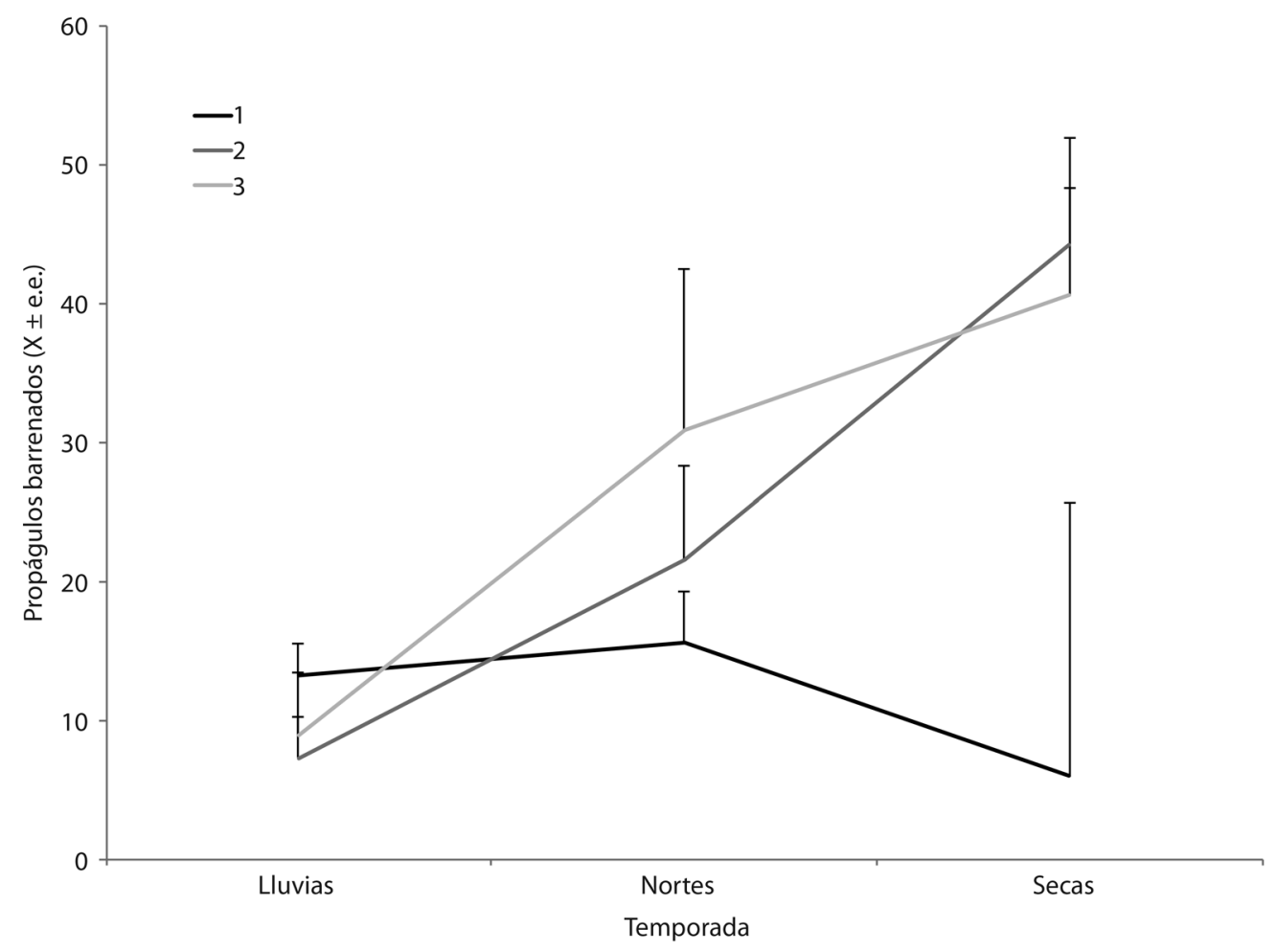

Fig. 1. Propágulos de $R$. mangle barrenados por $C$. rhizophorae en las tres zonas evaluadas $(1=R$. mangle, $2=R$. mangle y $A$. germinans, $3=T$. latifolia y $R$. mangle) durante las temporadas de lluvias, nortes y secas.

Fig. 1. Propagules of $R$. mangle bored by $C$. rhizophorae at the three zones evaluated $(1=R$. mangle, $2=R$. mangle and A. germinans, $3=T$. latifolia and $R$. mangle) during rainy, north winds and dry seasons.

la posición del daño donde fue barrenado el propágulo $\left(\chi^{2}=17.10, \mathrm{p}<0.001\right)$, la zona $\left(\chi^{2}\right.$ $=30.54, \mathrm{p}<0.001)$, la temporada $\left(\chi^{2}=7.57, \mathrm{p}\right.$ $=0.023)$, así como las interacciones de zona y posición del daño $\left(\chi^{2}=21.18, \mathrm{p}<0.001\right)$ y la de zona y temporada $\left(\chi^{2}=29.33, \mathrm{p}<0.001\right)$.

Según la posición del daño, la supervivencia de los propágulos fue mayor cuando fueron barrenados en la posición basal o media, a diferencia de cuando fueron barrenados en la parte apical (apical $=0.15 \pm 0.06$, medio $=0.49$ \pm 0.08 , basal $=0.60 \pm 0.05$ ). Sin embargo, los escolítidos perforaron en su mayoría la parte basal del propágulo (basal $=80 \%$, medio $=12$ $\%$ y apical $=7 \%$ ). En función de la zona, la 1 presentó una menor proporción de supervivencia; mientras que en la zona 2 y 3 se observó la mayor proporción de supervivencia de propágulos barrenados $(1=0.30 \pm 0.07,2=0.50$ $\pm 0.08,3=0.44 \pm 0.06)$. No obstante, la zona 2 presentó una menor frecuencia de propágulos barrenados con respecto a la zona 1 y $3(1=$ $37 \%, 2=18 \%$ y $3=45 \%$ ). Por temporada, las secas y nortes albergaron mayor proporción de propágulos barrenados vivos que durante las lluvias (lluvias $=0.34 \pm 0.08$, nortes $=0.45 \pm$ 0.07 , basal $=0.45 \pm 0.07$ ); la mayor frecuencia de propágulos en este caso se observaron en secas y lluvias (lluvias $=15 \%$, nortes $=36 \%$, secas $=48 \%$ ).

De las interacciones del GLM, observamos que en las zonas 1 y 2 hubo mayor número de propágulos vivos con daño en la posición basal, mientras que en la zona 3 la mayor supervivencia la presentaron los propágulos barrenados en la parte media (Fig. 2A). En la segunda 

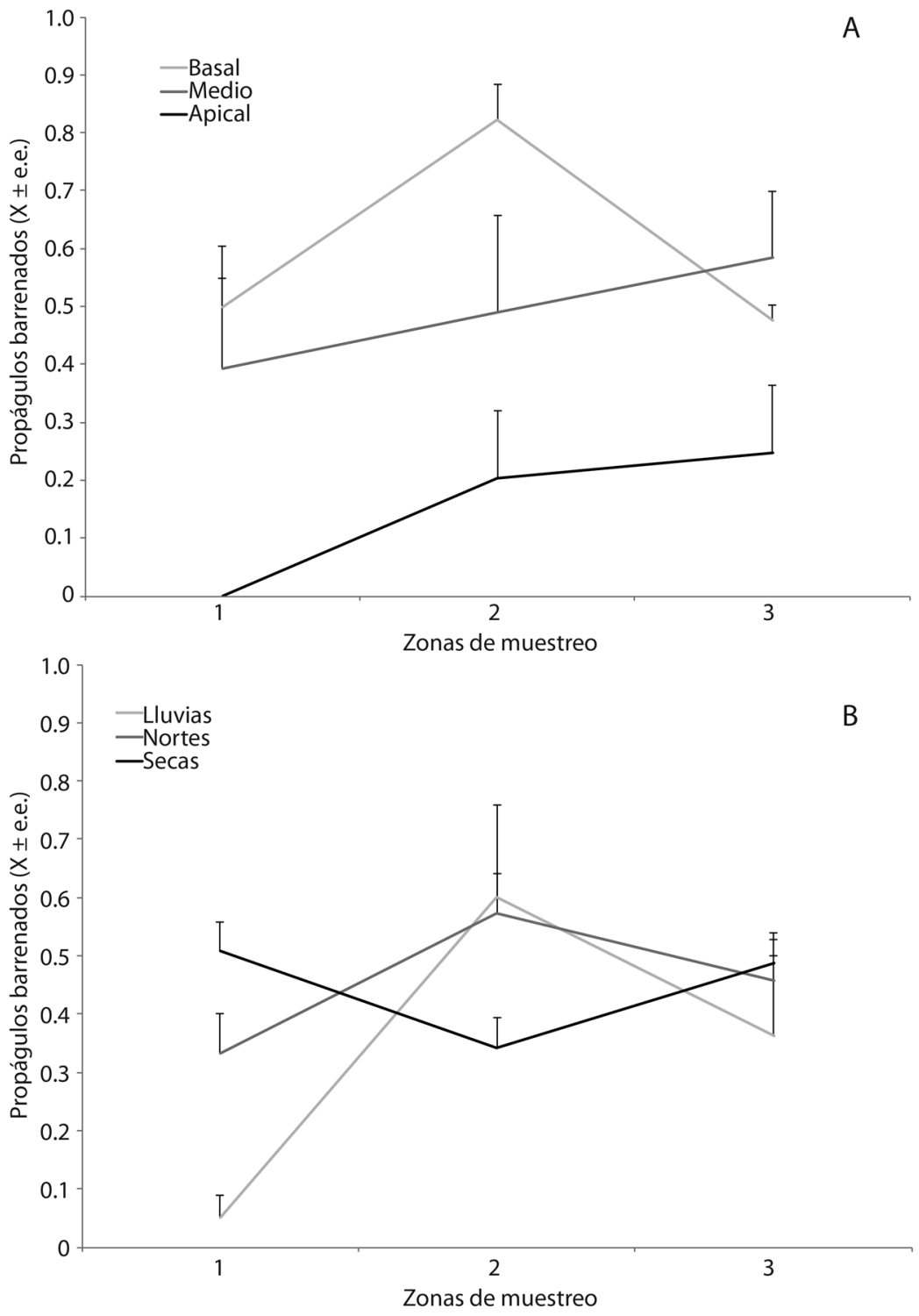

Fig. 2. Proporción de la supervivencia de propágulos barrenados: (A) interacción entre la posición del daño (Basal, medio y apical) y zonas muestreadas, y (B) interacción entre temporada (lluvias, nortes y secas) y zonas de muestreo.

Fig. 2. Proportion of surviving bored propagules: (A) interaction between position of the damage (basal, middle and apical) and the sampling zones, and (B) the interaction between the season (rainy, norte and dry) and the sampling zones.

interacción, se observó que las zonas se comportaron de manera diferente; la zona 1 mostró una supervivencia baja en lluvias y alta en secas, en la zona 2 la mayor proporción de propágulos vivos se presentó durante las lluvias y nortes, mientras que en la zona 3 las tres temporadas mostraron la misma proporción de supervivencia de propágulos barrenados (Fig. 2B).

Sobre la influencia de las variables fisicoquímicas, el nivel de inundación mostró una relación negativa con el número de propágulos barrenados por C. rhizophorae en el manglar de Tumilco. El número de propágulos disminuyó 
conforme los niveles de inundación fueron mayores $(r=0.524, p=0.0176)$. Con respecto a las variables salinidad, $\mathrm{pH}$, precipitación, temperatura y humedad, no se observó ninguna correlación $(\mathrm{r}=0.175, \mathrm{p}=0.228, \mathrm{r}=0.014, \mathrm{p}=$ $0.929, \mathrm{r}=0.015, \mathrm{p}=0.971, \mathrm{r}=0.105, \mathrm{p}=0.473$, $\mathrm{r}=0.739, \mathrm{p}=0.093$, respectivamente).

\section{DISCUSIÓN}

Existe una gran diversidad de coleópteros que afectan las plantas silvestres (Wylie \& Speight, 2012). En el caso de los propágulos de $R$. mangle, se identificó que la especie que barrena los propágulos abortados en el manglar de Tumilco, Veracruz, es C. rhizophorae (Hopkins) (Coleoptera: Curculionidae: Scolytinae). Previamente, Atkinson y Equihua (1986) registraron $C$. rhizophorae en los manglares de Veracruz. De la misma forma, GerónimoTorres et al. (2015) lo encontraron en Tabasco junto con otras especies asociadas al manglar. A nivel internacional, C. rhizophorae ha sido registrado en manglares de diferentes países (Rabinowitz 1977; Maiti \& Saha, 1986; Murphy, 1990; Feller \& Mathis, 1997; Wood \& Bright, 1992; Castillo, 2001; Sousa et al., 2003a; Devlin, 2004; Menéndez et al., 2006). C. rhizophorae se reconoce como un parásito obligado de $R$. mangle y hasta ahora es el único coleóptero que se ha encontrado barrenando propágulos en el estado de Veracruz. Es importante mencionar que en propágulos muertos, perforados en el manglar de Tumilco, se ha encontrado la especie Acalles sablensis (Coleoptera: Curculionidae); no obstante, se considera que es una especie oportunista que puede aprovechar las galerías previamente fabricadas por C. rhizophorae (ChamorroFlorescano, López-Guillén, Martínez-Zacarías, \& OBrien, 2014).

En este estudio se observaron variaciones en el número de propágulos barrenados por $C$. rhizophorae en las temporadas de muestreo. Se registró una mayor frecuencia de propágulos barrenados durante la temporada de secas, seguida de nortes y con menor frecuencia en lluvias. Menéndez et al. (2006), en un estudio similar en Cuba, observaron que la frecuencia de propágulos barrenados por curculiónidos mensualmente aumentó en los meses de mayor pluviosidad. Los resultados en este estudio indican que existen diferencias estadísticamente significativas entre temporadas, en los meses de julio a octubre la frecuencia de propágulos barrenados disminuyó a diferencia del resto de los meses. Tovilla y Orihuela (2002) mencionan que durante la temporada de lluvias los propágulos son exportados hacia otras áreas, incluso a la zona marina, debido a que son removidos por la marea durante la inundación. En nuestro estudio, los propágulos pudieron ser removidos de la zona de estudio debido a la inundación en la temporada de lluvias, aunado a la tormenta Ernesto que se presentó a inicio de temporada y que provocó un incremento inusual en el nivel de la marea.

Según estudios previos, las zonas cerradas dentro del manglar registran un mayor número de propágulos barrenados que las zonas abiertas (Sousa et al., 2003b; Devlin, 2004), sin embargo, en este trabajo se encontró lo inverso. Se sabe que algunos escolítidos son atraídos por la luz (Browne, 1961), en este caso, en la zona 2 y 3 hay relativamente mayor entrada de luz. Sería importante en un futuro evaluar qué otros posibles parámetros ambientales asociados a zonas abiertas o cerradas en Tumilco favorecen que los propágulos de $R$. mangle sean barrenados.

En el manglar de Tumilco, la supervivencia de los propágulos barrenados varió de acuerdo a las variables analizadas. Onuf et al. (1977), mostraron que los propágulos barrenados por escolítidos disminuían su viabilidad. En este trabajo, se observó con mayor frecuencia el daño estructural generado por C. rhizophorae en la parte basal. Rabinowitz (1977), sugiere que los propágulos que son perforados en la parte basal pueden aún tener diferentes estrategias para el desarrollo de raíces y así solventar el daño al tejido. En este trabajo no se evaluó el desarrollo de raíces; sin embargo, se comprobó un incrementó en la supervivencia de los propágulos con daño en esta posición. Aunque con menor frecuencia se encontraron 
propágulos con daños en las partes media y apical, sería importante evaluar el microhábitat que genera el escolítido en el interior del propágulo y observar la relación entre posición y daño en el interior, y los mecanismos que tiene la planta para solventar el problema.

El núcleo del manglar (1), mostró una baja supervivencia de propágulos barrenados, mientras que la zona asociada a la vegetación emergente (3) y la zona al borde de la carretera (2) presentaron una mayor supervivencia de los propágulos barrenados. Sousa et al. (2003a), encontraron que los propágulos que estaban más propensos a ser barrenados a causa de C. rhizophorae, e incluso con menor supervivencia, eran los que se encontraban en zonas con dosel cerrado; similar a este estudio, los propágulos encontrados bajo una vegetación más cerrada tuvieron mayor porcentaje de propágulos muertos, contrario a la zona con mayor alteración, que fue el borde de la carretera.

La mayoría de los propágulos en las tres zonas muestreadas tenían el daño en la parte basal; sin embargo, solo en la zona 2, los propágulos sobrevivieron más con el daño en esta posición. Estos resultados pueden estar relacionados con los niveles de luz (Browne, 1961). Según estudios anteriores, bajos niveles de luz pueden producir estrés en las plantas, para $R$. mangle los niveles de luz representan una variable importante, así como para los nutrientes que se generan (Feller \& Sitnik, 1996; Sousa et al., 2003b). En la zona 2 hay mayor luminosidad, y aunque el daño del propágulo fuera en la parte basal, las condiciones favorecen la solución del daño y los propágulos tienen mayores probabilidades de sobrevivir.

Por otra parte, las zonas de muestreo en este estudio se establecieron donde el nivel de inundación tenía variaciones durante el día por efecto de la marea y en consecuencia, la humedad y la salinidad podían generar un estrés en los propágulos. Sin embargo, el nivel de inundación fue la única variable que mostró una relación positiva con la frecuencia de propágulos barrenados. Diversos estudios han mostrado que factores ambientales como salinidad, nivel de inundación y humedad pueden influir en la infestación de propágulos y plántulas en los manglares (Onuf et al., 1977; Sousa et al., 2003b; Devlin, 2004; Krauss et al., 2008). En este trabajo las variables evaluadas no fueron tomadas en cada uno de las zonas de muestreo, sino de una sola estación climática perteneciente a CONAGUA, por lo cual no se puede descartar que las variables consideradas influyan en el número de propágulos barrenados en el manglar de Tumilco. Se requiere de una evaluación más exhaustiva para observar la relación de los factores fisicoquímicos con la presencia de $C$. rhizophorae.

Se considera de gran relevancia investigar el daño que genera el escolítido en el establecimiento no solo de los propágulos, sino también de las plántulas en el manglar de Tumilco, para tener un panorama de cómo potencialmente C. rhizophorae puede afectar la estructura y la dinámica de $R$. mangle, ya que si los propágulos y plántulas sobreviven al daño, esto puede modificar su crecimiento y el desarrollo estructural de los árboles. Cabe mencionar que Rhizophora mangle esta categorizada como especie amenazada por la Norma Oficial Mexicana (2010) NOM-059-SEMARNAT 2010.

\section{AGRADECIMIENTOS}

A Mario E. Favila por los comentarios que ayudaron a mejorar el manuscrito, a Thomas Atkinson por la identificación del escolítido y a Blanca Raya por su apoyo en la edición de las figuras.

\section{RESUMEN}

Los propágulos de las especies de manglar presentan una particular estrategia de superviviencia y dispersión. Desafortunadamente algunos reportes han afirmado que los propágulos de Rhizophora mangle son frecuentemente atacados por coleópteros y lepidópteros antes y después de su período de dispersión. El escarabajo Coccotrypes rhizophorae es un parásito obligado que puede causar la mortalidad de propágulos y plántulas de $R$. mangle, moldeando la estructura de sus poblaciones. Debido a la importancia de esta especie de mangle, su sobrevivencia y el aumento del impacto humano en la zona, este trabajo evaluó la presencia de propágulos barrenados por C. rhizophorae en el manglar de Tumilco, en el estado de Veracruz, 
México. Para esto, se establecieron tres zonas de muestreo: 1) manglar de $R$. mangle, 2) zona más impactada al borde de un camino de terracería con $R$. mangle y A. germinans, y 3 ) borde con Typha latifolia y $R$. mangle; en cada área, tres parcelas aleatorias de $25 \times 25 \mathrm{~m}$ fueron analizadas. Se registró el número de propágulos barrenados por zona cada 15 días durante un año de muestreo $(n=567)$ de enero a diciembre 2012. También observamos el área dañada del propágulo (basal, medio, apical) y los propágulos barrenados vivos y muertos por temporada y los resultados para diferentes temporadas en el área: lluvias, nortes y secas. Los insectos fueron identificados por un experto. Nuestros resultados muestran un incremento en el número de propágulos barrenados durante la temporada de secas, principalmente en la zona 2 y 3 . La supervivencia de los propágulos barrenados fue mayor cuando se presentó la perforación en el área basal, y durante la temporada de secas y nortes. En particular, la zona de estudio 2 presentó mayor número de propágulos vivos cuando el daño se encontró en el área basal del propágulo. En las tres zonas hubo mayor mortalidad cuando el daño fue apical. La zona 1 y 3 presentaron mayor supervivencia de propágulos durante las secas, mientras que en la zona 2 la supervivencia fue mayor durante la temporada de lluvias y nortes. El número de propágulos disminuyó conforme los niveles de inundación aumentaron. Los resultados muestran que C. rhizophorae tiene una amplia distribución en el manglar de Tumilco, pero la mortalidad de los propágulos de $R$. mangle está infuenciada por el área dañada, por la temporada del año y por las características de la zona en donde se encuentre la infestación. Se requieren estudios para evaluar la forma en que la mortalidad de los propágulos afecta la estructura poblacional de $R$. mangle.

Palabras clave: supervivencia, escarabajo, depredación, mangle rojo, mortalidad.

\section{REFERENCIAS}

Atkinson, T. H., \& Equihua, M., A. (1986). Biology of bark and ambrosia beetles (Coleoptera: Scolytidae and Platypodidae) of a tropical rain forest in southeastern Mexico with an annotated checklist of species. Annals of the Entomological Society of America, 79, 414-423.

Basáñez-Muñoz, A. (2005). Ficha Informativa de los Humedales Ramsar (FIR). Gland, Suiza: Oficina de la Convención de Ramsar.

Bright, D. E., \& Peck, S. B. (1998). Scolytidae from the Galápagos Islands, Ecuador, with descriptions of four new species, new distribution records, and a key to species (Coleoptera: Scolytidae). Koleopterologische Rundschau, 68, 233-252.

Browne, F. G. (1961). The biology of Malayan Scolytidae and Platypodidae. Malayan Forest Records no. 22. Malaya: Government Press.
Cannicci, S., Burrows, D., Fratini, S., Smith, T. J., Offenberg, J., \& Dahdouh-Guebas, F. (2008). Faunal impact on vegetation structure and ecosystem function in mangrove forests: a review. Aquatic Botany, 89(2), 186-200.

Castillo, P. S. (2001). Evaluación de las principales plagas del "mangle rojo" (Rhizophora mangle), "mangle salado" (Avicennia germinans) y "mangle blanco" (Laguncularia racemosa) en Tumbes, Perú. Revista Peruana de Entomología, 42, 185-189.

Chamorro-Florescano, I. A., López-Guillén, G., MartínezZacarías, A. A., \& OBrien, C. W. (2014). First Record of Acalles sablensis (Coleoptera: Curculionidae) in Rhizophora mangle (Malpighiales: Rhizophoraceae) along the Gulf of Mexico. Florida Entomologist, 97(4), 1869-1872.

Crawley, M. J. (2013). The R Book. Chichester, UK: John Wiley \& Sons.

Devlin, D. J. (2004). Analyses of the relationship between a parasitic beetle (Coccotrypes rhizophorae) and a host plant, the red mangrove (Rhizophora mangle) (Ph. D. Dissertation). University of Lafayette, Lafayette, LA, USA.

Feller, I. C., \& Sitnik, M. (1996). Mangrove ecology: a manual for a field course. Washington, DC, USA: Smithsonian Institution.

Feller, I. C., \& Mathis, W. N. (1997). Primary herbivory by wood-boring insects along an architectural gradient of Rhizophora mangle. Biotropica, 29(4), 440-451.

Gerónimo-Torres, J. D. C., Pérez-De La Cruz, M., De La Cruz-Pérez, A., \& Torres-De La Cruz, M. (2015). Scolytinae y Platypodinae (Coleoptera: Curculionidae) asociados a manglares de Tabasco, México/ Platypodinae and Scolytinae (Coleoptera: Curculionidae) associated with mangroves in Tabasco, Mexico. Revista Colombiana de Entomología, 41(2), 257.

Krauss, K. W., Lovelock, C. E., McKee, K. L., LópezHoffman, L., Ewe, S. M., \& Sousa, W. P. (2008). Environmental drivers in mangrove establishment and early development: a review. Aquatic Botany, 89(2), 105-127.

Maiti, P. K., \& Saha, N. (1986). Contribution to the knowledge of the bark and timber beetles (Scolytidae, Coleoptera) of the islands of Andaman and Nicobar, India. Miscellaneous Publication, Occasional Paper, $86,1-182$.

Menéndez, L., Guzmán, J. M., \& Núñez, R. (2006). Aspectos de la relación planta animal en los manglares cubanos. En L. Menéndez \& J. M. Guzmán (Eds.), Ecosistema de manglar en el archipiélago cubano (pp. 235-242). Estudios y experiencias enfocados a su gestión. Academia, La Habana, Cuba: Editorial Academia. 
Murphy, D. H. (1990). The natural history of insect herbivory on mangrove trees in and near Singapore. Raffles Bulletin of Zoology, 38(2), 119-203.

Norma Oficial Mexicana. (2010). NOM-059ECOL-2001. Protección ambiental-Especies nativas de México de flora y fauna silvestres-Categorías de riesgo y especificaciones para su inclusión, exclusión o cambio. Lista de especies en riesgo. México: Diario Oficial de la Federación (Segunda sección).

Onuf, C. P., Teal, J. M., \& Valiela, I. (1977). Interactions of nutrients, plant growth and herbivory in a mangrove ecosystem. Ecology, 58(3), 514-526.

Rabinowitz, D. (1977). Effects of a mangrove borer, Poecilips rhizophorae, on propagules of Rhizophora harrisonii in Panamá. Florida Entomologist, 60, 129-134.

Rico-Gray, V. \& Palacios-Ríos, M. (1996). Salinidad y el nivel del agua como factores en la distribución de la vegetación en la ciénaga del NW de Campeche, México. Acta Botánica Mexicana, 34, 53-61.

R Development Core Team. (2014). R: A Language and Environment for Statistical Computing. R Foundation for Statistical Computing, Vienna, Austria. Recuperado de: http://www.R-project.org/.

Rodríguez-Zúñiga, M. T., Troche-Souza, C., Vázquez-Lule, A. D., Márquez-Mendoza, J. D., Vázquez-Balderas, B., Valderrama-Landeros, L., Velázquez-Salazar, S., Cruz-López, M. I., Ressl, R., Uribe-Martínez, A., Cerdeira-Estrada, S., Acosta-Velázquez, J., DíazGallegos, J., Jiménez-Rosenberg, R., Fueyo- Mac Donald, L. \& Galindo-Leal, C. (2013). Manglares de México/Extensión, distribución y monitoreo. México, DF: Comisión Nacional para el Conocimiento y Uso de la Biodiversidad.

Sánchez-Martínez, G., \& Wagner, M. R. (2002). Bark beetle community structure under four ponderosa pine forest stand conditions in northern Arizona. Forest Ecology and Management, 170(1), 145-160.

Sousa, P. W., Kennedy, G. P., \& Mitchell, J. B. (2003a). Propagule size and predispersal damage by insects affect establishment and early of mangrove seedlings. Oecologia, 135, 564-575.

Sousa, P. W., Quek, P. S., \& Mitchell, J. B. (2003b). Regeneration of Rhizophora mangle in Caribbean mangrove forest: interacting effects of canopy disturbance and a stem-boring beetle. Oecologia, 137, 436-445.

Souza, M. M., \& Sampaio, E. V. (2011). Predation on propagules and seedlings in mature and regenerating mangroves in the coast of Ceará, Brazil. Hydrobiologia, 661(1), 179-186.

Toledo-Ocampo, A. (2005). Marco conceptual: Caracterización ambiental del Golfo de México. En A. V. Botello, J. Rendón-von Osten, G. Gold-Bouchot \& C. Agraz-Hernández (Eds.), Golfo de México. Contaminación e impacto ambiental: Diagnóstico y tendencias (pp. 25-54). Campeche, México: Universidad Autónoma de Campeche, Universidad Nacional Autónoma de México, Instituto Nacional de Ecología.

Tovilla, H. C., \& Orihuela, D. E. (2002). Floración, establecimiento de propágalos y supervivencia de Rhizophora mangle L. en el manglar de Barra de Tecoanapa, Guerrero, México. Maderas y Bosques, 8, 89-102.

Vázquez Moreno, L. L., Rodríguez Pérez, M., \& Zorri1la, M. A. (2003). Lista de escolítidos (coleoptera) de Cuba y sus plantas hospedantes. Fitosanidad, $7(1), 17-23$.

Wood, S. L., \& Bright Jr, D. E. (1992). A catalogue of Scolytidae and Platypodidae (Coleoptera). Part 2, Volumes A \& B. Great Basin Naturalist Memoirs, 13, 1-1551.

Wood, S. L., Stevens, G. C., \& Lezama, H. J. (1991). Los Scolytidae de Costa Rica: Clave de géneros y de la subfamilia Hylesinae (Coleoptera). Revista de Biología Tropical, 39, 125-148.

Woodruff, R. E. (1970). A mangrove borer, Poecilips rhizophorae (Hopkins) (Coleoptera: Scolytidae). Entomology Circular, 98, 1-2.

Wylie, F. R., \& Speight, M. R. (2012). Insect pests in tropical forestry. London, UK: CABI. 